\title{
Microstructural Characterization of AICrTiV - Si High Entropy Alloy for advanced applications
}

\author{
Ioannis Daskalopoulos ${ }^{1}$, Spyridon Chaskis ${ }^{1 *}$, Marianthi Bouzouni ${ }^{2}$, Pavlos Stavroulakis ${ }^{2}$, \\ Russell Goodall ${ }^{2}$, Spyros Papaefthymiou ${ }^{1}$
}

${ }^{1}$ Laboratory of Physical Metallurgy, Division of Metallurgy and Materials, School of Mining and Metallurgical Engineering, National Technical University of Athens, 9, Her. Polytechniou Str., Zografos, 15780 Athens, Greece

${ }^{2}$ Department of Physical Metallurgy and Forming, Hellenic Research Centre for Metals (ELKEME S.A.), $61 \mathrm{st} \mathrm{km}$ Athens-Lamia Nat. Road, Oinofyta, 32011 Viotia, Greece

${ }^{3}$ Department of Material Science \& Engineering, The University of Sheffield, Sir Robert Hadfield Building, Mappin St, Sheffield, S13 JD, United Kingdom

\begin{abstract}
This work deals with the microstructural characterization of two equiatomic high-entropy, low-density alloys (HEA), the AlCrTiV and AlCrTiV-Si ${ }_{7.2}$. These alloys can serve as potential candidates for advanced applications where high strength and enhanced ductility is demanded. For ensuring high ductility the alloys must contain as minimum as possible hard precipitates. As the strength increase is based on both solid solution and precipitation hardening, the laboratory made alloys were investigated in ascast and heat-treated conditions. For the heat treatment a high soaking temperature of $1200^{\circ} \mathrm{C}$ for 8 hours was selected to ensure microstructure homogenization. Micrographic observations of the AlCrTiV and AlCrTiVSi7.2 samples in the as-cast condition indicated the presence of a dendritic microstructure. Furthermore, chemical micro-analysis showed segregation in the matrix in both samples. This is a critical result as this segregation will lead to heavy precipitation at interdendritic regions, it may sensitize these regions and in the worst-case scenario may cause cleavage fracture in the micro scale, which can trigger brittle fracture during cooling even without the application of deformation. However, the selected heat treatment eliminated the segregation phenomena forcing the alloying elements to be uniformly distributed in the matrix. At the center of the heat-treated AlCrTiV-Si 7.2 sample the fragmentation and spheroidization of the intermetallic phase $\mathrm{Ti}_{5} \mathrm{Si}_{3}$ was observed. For the same sample, at the moldsample's interface, the particles $\mathrm{Ti}_{5} \mathrm{Si}_{3}$ were shown to dissolve and form aggregates. Both alloys exhibited high hardness values with small differences between the as-cast and heat-treated conditions, which indicates that the AlCrTiV-Si7.2 high entropy alloy presents high yield strength and may operate at high temperatures without deterioration of the mechanical properties nor unexpected failure.
\end{abstract}

* Corresponding author: s.chaskis@metal.ntua.gr 


\section{Introduction}

Currently, there are 2 widely used ways in order to process what a High-Entropy Alloy (HEA) is. The first theory, categorizes HEAs as alloys, which contain at least 5 main alloying elements varying from 5 to 35 at. $\%$ and potential additional elements below 5 at. \%. The second one is based on the configurational entropy and accepts HEAs as alloys which have:

Where: $\Delta$ Sconf. $=$ configurational entropy.

$$
\Delta \mathrm{S}_{\text {conf }}>1.5 \mathrm{R}
$$

$$
\Delta \mathrm{S}_{\text {conf }}=-\mathrm{R} \sum_{\mathrm{i}=1}^{\mathrm{n}} \mathrm{c}_{\mathrm{i}} \ln \left(\mathrm{c}_{\mathrm{i}}\right)
$$

Where: $\mathrm{R}=8.314 \mathrm{~J} / \mathrm{K}$ mol (gas constant)

$\mathrm{ci}=$ atomic concentration of element $\mathrm{i}[1]$.

In the recent years a new category of lightweight high-entropy alloys (LWHEAs) has emerged with the aim of creating materials with high specific mechanical properties. The AlCrTiV and AlCrTiV-Sis alloys are distinct examples of successful HEA alloy design, by combining characteristics from both the LWHEAs and refractory high-entropy alloys (RHEAs) categories [2]. The alloys produced in this study can be considered as MediumEntropy Alloys (MEAs)

$$
1 R \leq \Delta \mathrm{S}_{\mathrm{conf}} \leq 1.5 R
$$

Although, in the existing literature it is mentioned as HEA [2][3]. The equiatomic AlCrTiV is characterized by a single BCC (B2) solid solution, with relatively low density at $5.06 \mathrm{~g} / \mathrm{cm}_{3}$ and hardness value at $498 \mathrm{HV}$ [3]. Microalloying into the AlCrTiV system with silicon leads to the formation of a dual-phase microstructure, consisting of the intermetallic phase $\mathrm{Ti}_{5} \mathrm{Si}_{3}$ dispersed inside the matrix of the $\mathrm{BCC}$ (B2) solid solution. In BCC systems the most common structures are BCC_A2 and BCC_B2. Where A2 refers to disordered $\mathrm{BCC}$, while B2 to ordered (semi-ordered) BCC [4][5][6]. Thermodynamical calculations based on TCHEA2 database reveal the possibility of a transformation, which is referred as "order-disorder" transformation and it seems to occur at $700{ }^{\circ} \mathrm{C}(973 \mathrm{~K})$, as shown in X. Huang et. al. research work [7]. Above $700{ }^{\circ} \mathrm{C}$, the existing parent phase is BCC_A2, while below $700{ }^{\circ} \mathrm{C}$, it is transformed into BCC_B2. The theoretical density of the AlCrTiV-Si $i_{5}$ alloy is calculated between 4.7-4.9 $\mathrm{g} / \mathrm{cm}^{3}$ and its microhardness reaches the exceptionally high value of 679 $\mathrm{HV}_{0.2}$ Vickers hardness [7]. The combination of high hardness and low density, in addition to relatively available constituent alloying elements, highlights the need for further research on the AlCrTiV-Si system [8]. In this research the effects of heat treatment of the AlCrTiV and $\mathrm{AlCrTiV}-\mathrm{Si}_{7.2}$ alloys at $1200{ }^{\circ} \mathrm{C}$ for 8 hours were studied.

\section{Experimental Approach}

Two alloys were produced with the Vacuum Arc Melting (VAR) technique [6] and both were re-melted 5 times in combination with electromagnetic stirring, in order to achieve a fully homogenized microstructure. The first one is the Al-Ti-V-Cr and the other is the microalloyed Al-Ti-V-Cr-Si $i_{7.2}$. The two alloys underwent heat treatment at $1200{ }^{\circ} \mathrm{C}$ for 8 hours.

The results of the heat treatment were evaluated, by comparing the heat-treated samples with their as-cast counterparts. The samples were compared based on the presence of segregation phenomena, the characteristics of distribution of the particles and intermetallic phases and 
the samples microhardness values. Regarding means of metallography, samples underwent sectioning, grinding and polishing. For the microstructural characterization optical microscopy (OM) and electron microscopy/energy dispersive X-Ray spectroscopy (SEM/EDS) were utilized, while for the mechanical properties, microhardness measurements were conducted. The aspect ratio and mean diameter data of the intermetallic phases was generated by using OM measurements from central areas of the AlCrTiV-Si 7.2 samples and image analysis software. Moreover, the data was then processed with "ANOVA" using the Minitab18 software and MATLAB software.

\section{Results-Discussion}

\subsection{The Equiatomic Al-Cr-Ti-V system}

Regarding the AlCrTiV samples, in the as-cast condition the majority of the observable area with OM (Fig. 1) is characterized by a distinct dendritic microstructure and EDS maps (Fig. 2) shows segregation of the alloying elements inside the matrix, especially of Ti and V. The average microhardness value was calculated at $633 \mathrm{HV}_{0.2}$. In the respective heat-treated sample, the previously mentioned dendritic microstructure in the as-cast condition is replaced by coarse grains (Fig. 1). Results from EDS analysis showcase the total elimination of segregation in the matrix (Fig. 2) and the average microhardness is slightly decreased at a value of $619 \mathrm{HV}_{0.2}$, compared to the as-cast AlCrTiV. Based on the above results the heat treatment at $1200{ }^{\circ} \mathrm{C}$ for the AlCrTiV alloy has positive effects, by providing uniform distribution of the alloying elements and promotes grain development, while at the same time there is very little effect on the hardness values.

As-cast AlCrTiV

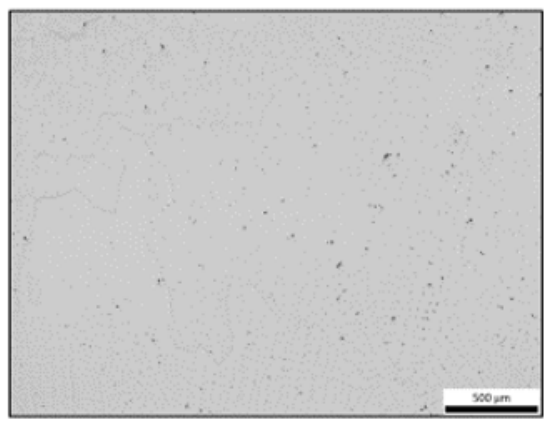

Heat-treated AICrTiV

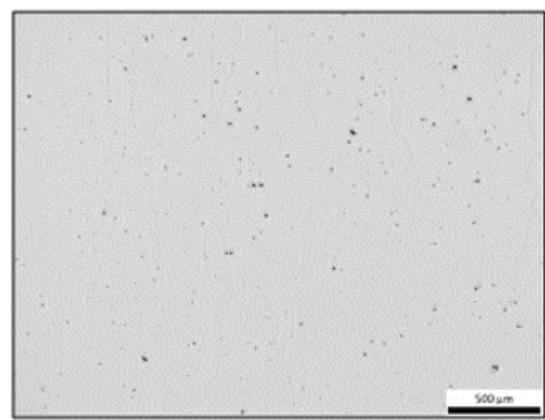

Fig. 1: Optical microscope micrographs from the centre of the AlTiVCr samples. 

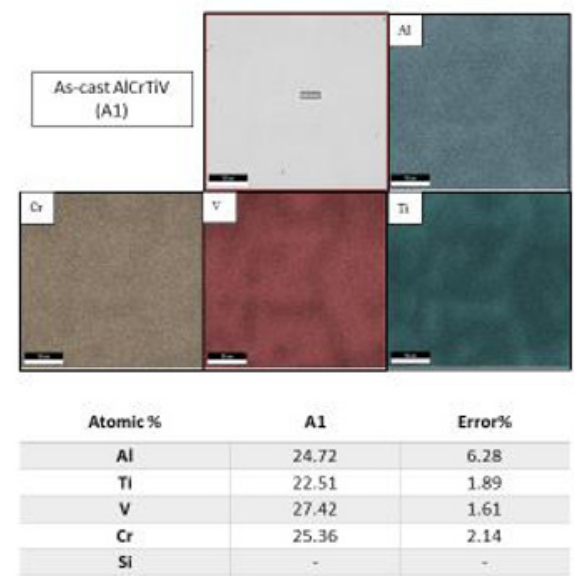
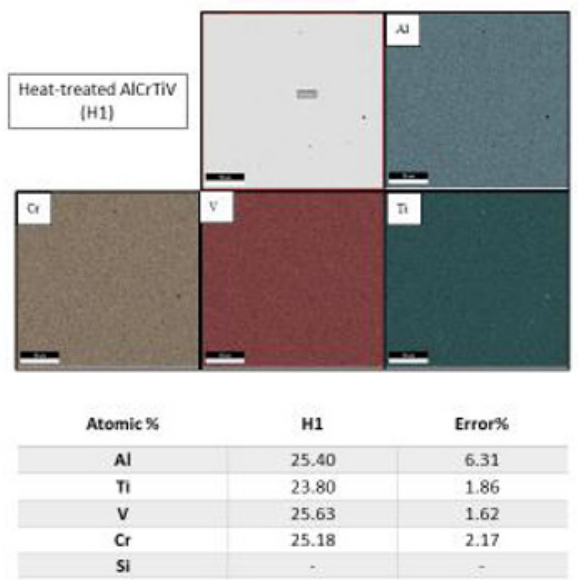

Fig. 2:EDS maps and elemental analysis of the AlCrTiV samples.

\subsection{The Al-Cr-Ti-V-Si $i_{7.2}$ system}

For the as-cast AlCrTiV-Si ${ }_{7.2}$ sample, much like the as-cast AlCrTiV, OM observations reveal a dendritic microstructure. Results from OM and SEM show elongated and sharp intermetallic phases at the centre of the sample (Fig. 3 and Fig. 5) and particles at the moldsample interface (Fig. 4 and Fig. 5). Segregation of the alloying elements inside the matrix is observed with EDS maps (Fig. 6). The average microhardness of the alloy is $760 \mathrm{HV}_{0,2}$. On the contrary, based on the EDS maps (Fig. 6) the heat-treated AlCrTiV-Si ${ }_{7.2}$ is shown to have an obvious lack of segregation. Observations with OM (Fig. 3) and SEM (Fig. 6) showcase the fragmentation and spheroidization of the intermetallic phase $\mathrm{Ti}_{5} \mathrm{Si}_{3}$ at the center of the sample, whereas the particles at the mold-sample interface have been dissolved and formed aggregates (Fig. 4 and Fig. 5), since the surface energy is higher. Both of these changes in the characteristics of $\mathrm{Ti}_{5} \mathrm{Si}_{3}$ could be contributed to the Ostwald Ripening phenomenon.
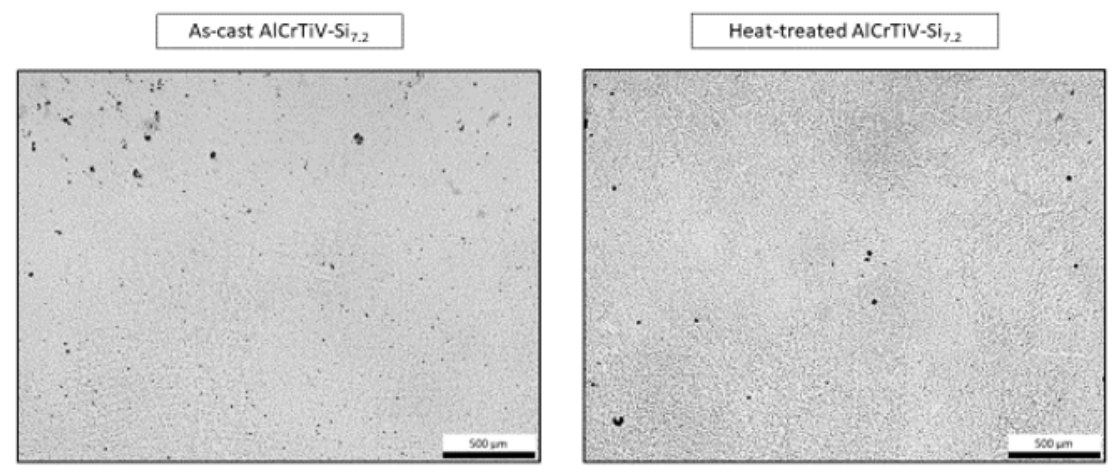

Fig. 3: Optical microscope micrographs from the centre of the AlTiVCr-Si7.2 samples. 

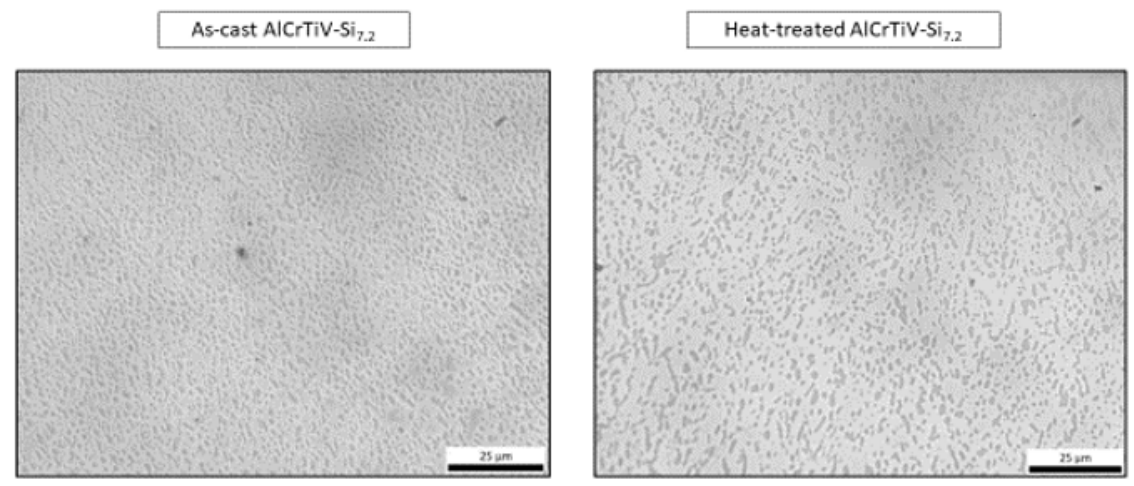

Fig. 4: Optical microscope micrographs from the mold-sample interface of the AlTiVCr-Si7.2 samples.

(As-cast AlCrTiV-Si7.2)

Mold-sample interface
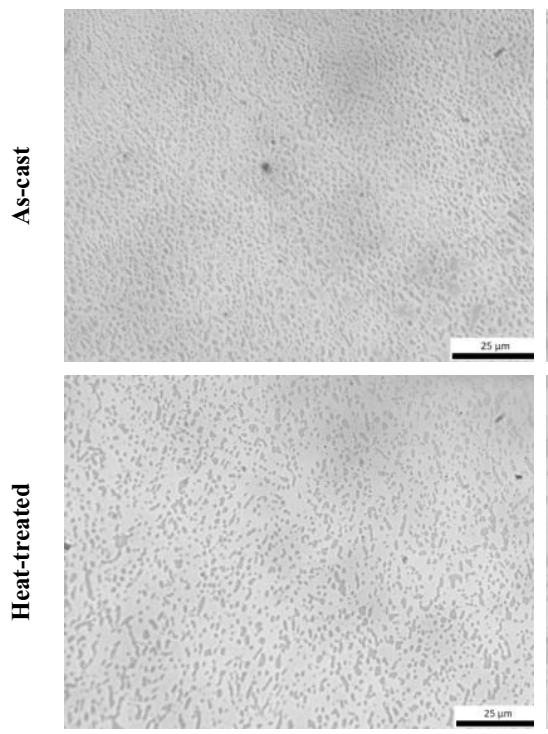

(Heat-treated AlCrTiV-Si7.2)

Center
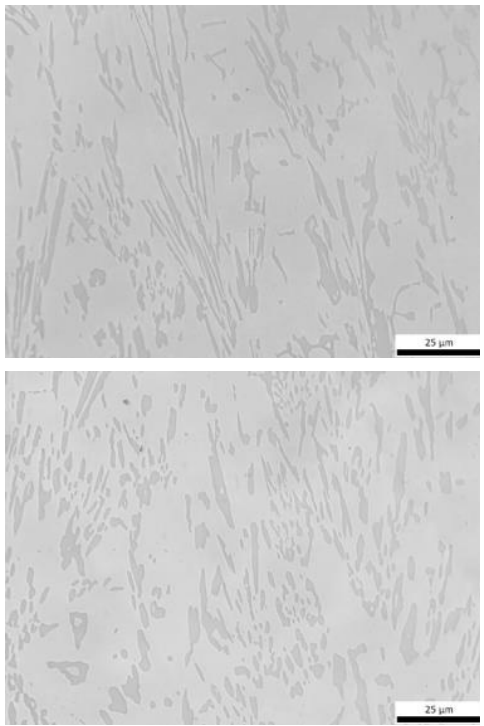

Fig. 5: Optical microscope micrographs (x1000) from the centre and mold-sample interface of the AlCrTiV-Si7.2 samples 

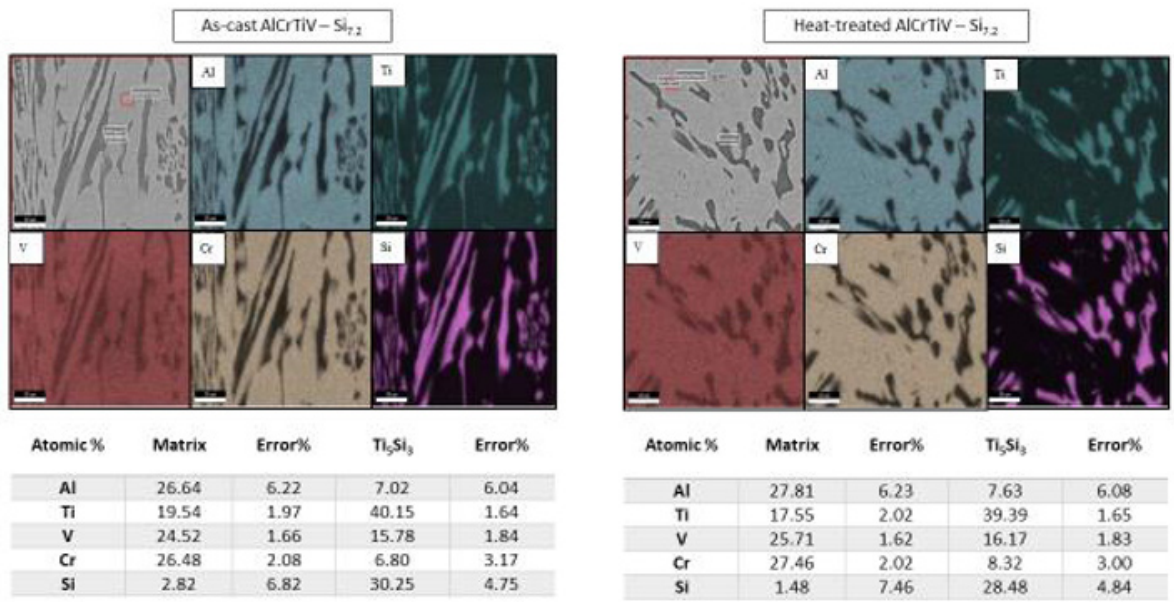

Fig. 6: EDS maps and elemental analysis of the AlTiVCr-Si7.2 samples.

\subsection{Discussion}

After statistical analysis with ANOVA (Fig. 7) and MATLAB (Fig. 8), the given results generated by using OM measurements support the previous statements. The decrease of the aspect ratio and mean diameter (Fig. 7) of the intermetallic phases in the heat-treated AlCrTiV-Si ${ }_{7.2}$, in comparison to the as-cast sample, validate the previous observations about spheroidization phenomena.
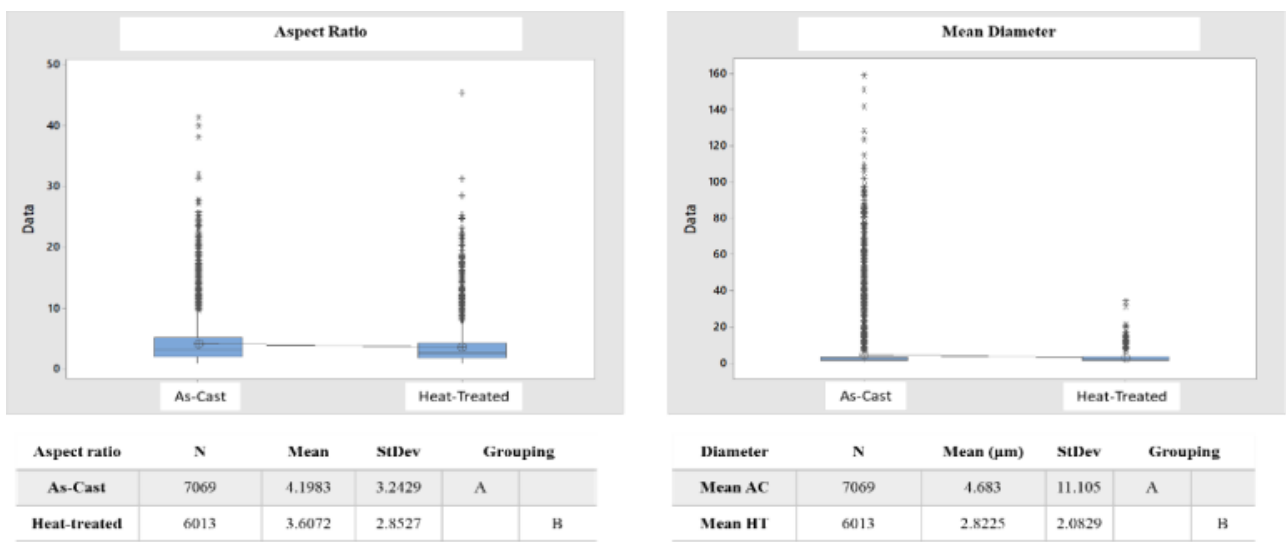

Fig. 7: ANOVA results of the Aspect Ratio and Mean Diameter of the $\mathrm{Ti}_{5} \mathrm{Si}_{3}$ intermetallic phases from the AlTiVCr-Si7.2 samples.

The decrease and convergence between the average and median values of the mean diameter in the heat-treated sample (Fig. 8), compared to the as-cast sample (Fig. 8), exhibits the tendency of the intermetallic phases to develop uniform characteristics, and thus reinforcing the theory behind the presence of the Ostwald Ripening phenomenon. 

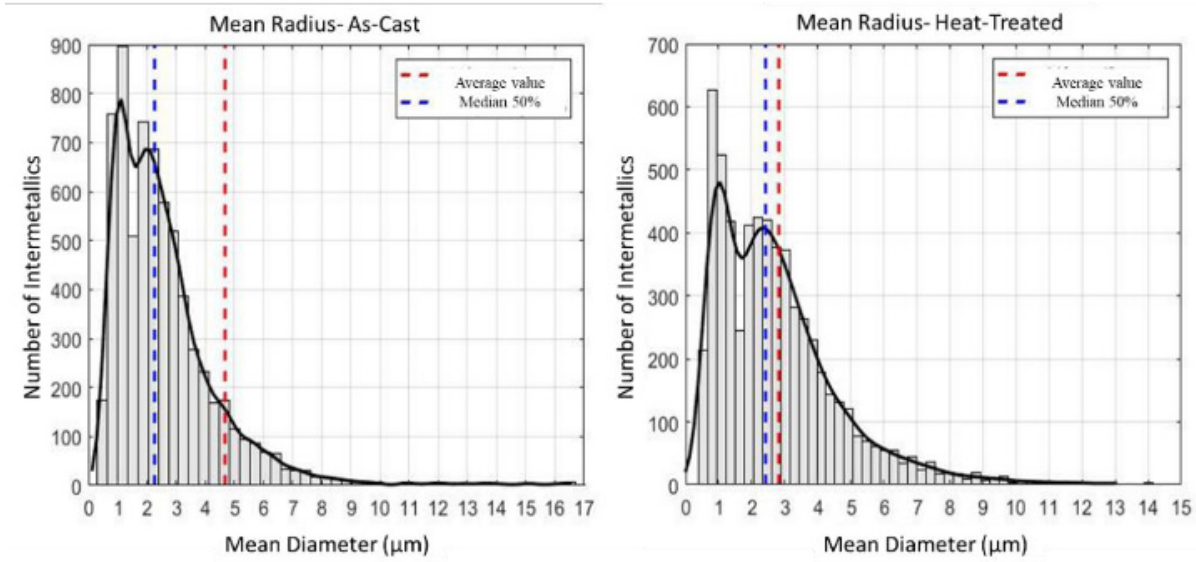

Fig. 8: MATLAB results for the mean diameter of the AlCrTiV-Si7.2 samples.

Finally, a small decrease in the average microhardness value is observed at $733 \mathrm{HV}_{0,2}$. From the above results, the heat-treatment of $\mathrm{AlCrTiV}-\mathrm{Si}_{7.2}$ at $1200{ }^{\circ} \mathrm{C}$ can be characterized as successful, hence there is a total elimination of segregation phenomena and uniform elemental distribution.

\section{Conclusions}

In this study the microstructure of the equiatomic AlCrTiV and AlCrTiV-Si 7.2 low-density HEAs was investigated in the as-cast and heat-treated condition after homogenization at 1200 ${ }^{\circ} \mathrm{C}$ for 8 hours. Observations of the AlCrTiV and AlCrTiV-Si ${ }_{7.2}$ samples in the as-cast condition indicated the presence of a dendritic microstructure, whereas elemental analysis results showed segregation in the matrix phase, in both samples. In the heat-treated condition both alloys exhibited an absence of segregation phenomena and instead showcased uniform distribution of the alloying elements in their matrixes.

At the centre of the heat-treated $\mathrm{AlCrTiV}-\mathrm{Si}_{7.2}$ sample the fragmentation and spheroidization of the intermetallic phase $\mathrm{Ti}_{5} \mathrm{Si}_{3}$ was observed. For the same sample at the mold-sample interface the particles of $\mathrm{Ti}_{5} \mathrm{Si}_{3}$ were shown to dissolve and form aggregates, as a result of the Ostwald Ripening phenomenon mechanism. The above results were evaluated with statistical modelling, which displayed that after heat treatment the intermetallic phase tends to embrace uniform characteristics. Both alloys exhibited high hardness values with small differences between the as-cast and heat-treated conditions.

Based on the above results, it is evident that the AlCrTiV and AlCrTiV-Si 7.2 alloys are susceptible to isothermal heat treatment at a high temperature. Further experiments are required in order to evaluate the effects of variation in heat treatment on the microstructure and the mechanical properties on both alloying systems.

\section{References}

[1] Y. Zhang, Y. J. Zhou, J. P. Lin, G. L. Chen, and P. K. Liaw, "Solid-Solution Phase Formation Rules for Multi-component Alloys," Adv. Eng. Mater., vol. 10, no. 6, pp. 534-538, Jun. 2008, doi: 10.1002/adem.200700240. 
[2] Y. Qiu et al., "A lightweight single-phase AlTiVCr compositionally complex alloy," Acta Mater., vol. 123, pp. 115-124, Jan. 2017, doi:

10.1016/j.actamat.2016.10.037.

[3] Y. Qiu, S. Thomas, M. A. Gibson, H. L. Fraser, K. Pohl, and N. Birbilis, "Microstructure and corrosion properties of the low-density single-phase compositionally complex alloy AlTiVCr," Corros. Sci., vol. 133, no. October 2017, pp. 386-396, 2018, doi: 10.1016/j.corsci.2018.01.035.

[4] A. K. Singh and A. Subramaniam, "On the formation of disordered solid solutions in multi-component alloys," J. Alloys Compd., vol. 587, pp. 113-119, 2014, doi: 10.1016/j.jallcom.2013.10.133.

[5] W. Sun, X. Huang, and A. A. Luo, "Phase formations in low density high entropy alloys," Calphad Comput. Coupling Phase Diagrams Thermochem., vol. 56, no. September 2016, pp. 19-28, 2017, doi: 10.1016/j.calphad.2016.11.002.

[6] S. Laube et al., "Controlling crystallographic ordering in $\mathrm{Mo}-\mathrm{Cr}-\mathrm{Ti}-\mathrm{Al}$ high entropy alloys to enhance ductility," J. Alloys Compd., vol. 823, p. 153805, 2020, doi: $10.1016 /$ j.jallcom.2020.153805.

[7] X. Huang, J. Miao, and A. A. Luo, "Lightweight AlCrTiV high-entropy alloys with dual-phase microstructure via microalloying," J. Mater. Sci., vol. 54, no. 3, pp. 2271-2277, Feb. 2019, doi: 10.1007/s10853-018-2970-4.

[8] A. Kumar and M. Gupta, "An insight into evolution of light weight high entropy alloys: A review," Metals (Basel)., vol. 6, no. 9, 2016, doi: 10.3390/met6090199. 